\title{
RISCO CARDIOMETABÓLICO ASSOCIADO À MEDIDAS ANTROPOMÉTRICAS EM FUNCIONÁRIOS DE UMA INSTITUIÇÃO DE ENSINO SUPERIOR DO INTERIOR DA PARAÍBA
}

\author{
CARDIOMETABOLIC RISK ASSOCIATED WITH ANTHROPOMETRIC \\ MEASURES IN EMPLOYEES OF A HIGHER EDUCATION \\ INSTITUTION IN THE INTERIOR OF PARAIBA
}

Daniel Pinheiro Fernandes ${ }^{1}$ Carolina Moreira de Santana ${ }^{2}$

Rayanne de Araújo Torres ${ }^{3}$

RESUMO: OBJETIVO: Avaliar o risco cardiometabólico de funcionários de uma instituição de ensino superior do interior da Paraíba a partir de dados antropométricos. MÉTODOS: Trata- se de um estudo transversal e quantitativo, realizado com funcionários de uma instituição de ensino superior da cidade de Cajazeiras - PB. Os elementos necessários para avaliar o risco cardiometabólico foram obtidos a partir de uma ficha clínica que continha campos relacionados à estilo de vida, histórico familiar e aos indicadores antropométricos (IMC, CC, RCQ e RCE). Após a coleta, os dados foram agrupados, tabulados e analisados por meio de estatística descritiva utilizando o Microsoft $\circledast$ Office Excel e apresentados em gráficos e tabelas. RESULTADOS: Os resultados mostraram uma elevada prevalência de fatores de risco cardiometabólico em ambos sexos. Em relação ao IMC, 18 indivíduos apresentaram obesidade, 20 foram classificados com risco muito elevado, segundo a CC, já em relação a RCQ e RCE, 12 e 31 participantes, respectivamente, apresentaram risco elevado. Em relação aos dados comportamentais dos avaliados, observou-se a prevalência de alguns hábitos que aumentam 0 risco cardiometabólico, como o consumo de álcool e a baixa prevalência da prática de atividade física. CONCLUSÃO: O presente trabalho foi capaz de evidenciar o risco cardiometabólico dos funcionários a partir de indicadores antropométricos e de forma suplementar, pelos fatores comportamentais. Assim, o estudo deve contribuir

\footnotetext{
${ }_{1}^{1}$ Acadêmico do curso de Bacharelado em Nutrição da Faculdade Santa Maria, Cajazeiras-PB. E-mail: dpinheiro15@yahoo.com.br.

${ }^{2}$ Nutricionista. Mestre em Sistema Agroindustriais pela UFCG. Docente do curso de Nutrição da Faculdade Santa Maria - FSM.

${ }^{3}$ Nutricionista. Doutora em Ciências da Nutrição pela UFPB. Coordenadora e docente do curso de Nutrição da Faculdade Santa Maria - FSM.
} 
para a formulação de medidas de intervenção nutricional e consequentemente, melhora da qualidade de vida e redução do risco cardiometabólico dos funcionários.

Palavras chave: Antropometria. Doenças cardiovasculares. Saúde do trabalhador. Síndrome metabólica.

ABSTRACT: OBJECTIVE: To assess the cardiometabolic risk of employees of a higher education institution in the interior of Paraiba using anthropometric data. METHODS: This is a cross-sectional and quantitative study, carried out with employees of a higher education institution in the city of Cajazeiras - PB. The elements necessary to assess cardiometabolic risk were used based on a clinical record that contains fields related to lifestyle, family history and anthropometric indicators (BMI, WC, WHR and WHR). After collection, the data were grouped, tabulated and analyzed using descriptive statistics using Microsoft ${ }^{\circ}$ Office Excel and displayed in graphs and tables. RESULTS: The results show a high prevalence of cardiometabolic risk factors in both sexes. Regarding BMI, 18 were obese, 20 were classified as having a very high risk, according to the CC, in relation to WHR and WHR, 12 and 31 participants, respectively, with high risk. Regarding the behavioral data of those evaluated, there was a prevalence of some factors that increase or increase cardiometabolic risk, such as alcohol consumption and the low prevalence of physical activity. CONCLUSION: The present work was able to demonstrate the employees' cardiometabolic risk from anthropometric indicators and in a supplementary way, due to behavioral factors. Thus, the study should contribute to the reduction of nutritional intervention measures and, consequently, to the improvement of quality of life and the reduction of employees' cardiometabolic risk.

Keywords: Anthropometry. Cardiovascular diseases. Occupational Health. Metabolic syndrome. 


\section{INTRODUÇÃO}

As doenças cardiovasculares (DCVs) representam um grupo de diversos distúrbios interligados, tais como aterosclerose, hipertensão arterial, insuficiência cardíaca (IC), doença vascular periférica, doença cardíaca isquêmica, doença coronariana, doença cerebrovascular, trombose venosa profunda e cardiopatia congênita. Esse grupo de doenças reflete um problema de saúde pública mundial, sendo considerado a principal causa de morte em todo o mundo. Estima-se que cerca de 17,7 milhões de pessoas morreram em decorrência de eventos cardiovasculares em 2015, esse valor representa cerca de $31 \%$ dos óbitos em todo o mundo no mesmo ano (OPAS, 2017; RAYMOND; COUCH, 2018).

Um dos principais fatores de risco envolvidos na patogênese desse grupo de doenças é a obesidade, pois o excesso de tecido adiposo, sobretudo na região visceral, se relaciona diretamente com o risco cardiometabólico, aumentando a incidência de doença coronariana, hipertensão, acidente vascular encefálico, diabetes tipo 2 e Síndrome Metabólica (SM). Esse tipo de acúmulo de gordura gera alterações metabólicas que afetam a saúde do coração, tais como hipertensão arterial, intolerância à glicose, aumento de marcadores pró-inflamatórios, disfunção endotelial e dislipidemias (LYSEN; ISRAEL, 2018; RAYMOND; COUCH, 2018).

A SM também se constitui como importante fator de risco cardiometabólico, caracterizada pela combinação de no mínimo três das seguintes alterações fisiopatológicas: obesidade visceral, hipertrigliceridemia, hipertensão arterial, hiperglicemia de jejum e alterações anormais nos níveis das lipoproteínas de alta e baixa densidade, HDL e LDL, respectivamente. Essas alterações estão associadas à uma maior prevalência de eventos cardiovasculares, como infarto agudo do miocárdio (IAM) e acidente vascular cerebral (AVC) (MARTINI; BORGES; GUEDES, 2014; BARROSO et al., 2017).

Dessa forma, pode-se destacar o papel dos indicadores antropométricos associados à obesidade e a SM como preditores de risco cardiometabólico, com 
destaque para o Índice de Massa Corporal (IMC), que pode identificar o excesso ou o déficit de peso e a Circunferência da cintura (CC), que quando elevada representa um fator de risco independente de doenças cardiovasculares. Além desses, a razão cintura-quadril $(R C Q)$ e a razão cintura-estatura (RCE), são medidas que quando fora dos padrões estabelecidos, podem representar risco cardiometabólico (LITCHFORD, 2018).

Além das alterações clínicas e antropométricas o risco cardiometabólico está associado à fatores comportamentais, tais como dietas inadequadas, estresse, sono insuficiente, sedentarismo e o consumo excessivo de álcool. Esses fatores influenciam diretamente a saúde cardiovascular, pois se manifestam através de eventos como elevação da pressão arterial, hiperglicemia, hipertrigliceridemia e o aumento do peso. Tem se visto que inúmeros eventos coronarianos podem ser evitados através da adoção de hábitos de vida mais saudáveis, como ter uma dieta saudável, exercitar-se regularmente, controlar o peso e abandonar o tabagismo (RAYMOND; COUCH, 2018).

As doenças cardíacas impõem, na maioria das vezes, limitações físicas, sociais, financeiras e de saúde que interferem na qualidade de vida dos indivíduos. Além disso, essas doenças impactam a sociedade negativamente, pois representam maiores despesas com tratamento, custos para fornecimento de assistência formal e informal, perda da produtividade no emprego e do bem-estar geral (STEVENS et al., 2018). Diante do exposto, o presente trabalho teve como objetivo avaliar o risco cardiometabólico associado à parâmetros antropométricos em funcionários de uma instituição de ensino superior do interior da Paraíba, a fim de determinar o grau de risco de desenvolvimento de DCVs e outras comorbidades.

\section{METODOLOGIA}

Trata-se de uma pesquisa do tipo descritiva, observacional com delineamento transversal e abordagem quantitativa. Realizada na Faculdade Santa Maria da cidade de Cajazeiras-PB, uma Instituição de Ensino Superior situada no alto sertão 
paraibano e com tempo de serviço de mais de 15 anos. A instituição funciona durante os turnos manhã, tarde e noite e aos finais de semana oferece cursos de pós-graduação nas diferentes áreas do ensino. Os dados do estudo são parte do projeto de pesquisa intitulado: "DIAGNÓSTICO DO RISCO CARDIOMETABÓLICO, ACOMPANHAMENTO NUTRICIONAL E AVALIAÇÃO DO IMPACTO DE ATIVIDADES EDUCATIVAS EM FUNCIONÁRIOS DE UMA INSTITUIÇÃO DE ENSINO SUPERIOR".

A amostra utilizada para o estudo foi selecionada da população representada pelos funcionários da Faculdade Santa Maria (FSM), que atendessem aos seguinte critérios de inclusão: a) funcionários devidamente vinculados à FSM; b) funcionários de ambos sexos; c) faixa etária entre 20 e 60 anos; d) apresentasse capacidade cognitiva para responder ao questionário; e) estivesse em condições físicas que permitissem a aferição das medidas; f) concordaram em participar do estudo e assinar o Termo de Consentimento Livre e Esclarecido (TCLE). Como também foram definidos alguns critérios de exclusão, como: a) funcionários fora da faixa etária de 20 aos 60 anos; b) que estivesses em acompanhamento nutricional; c) que fizessem uso de medicamentos anti-hipertensivos ou hipoglicemiantes; d) mulheres gestantes; e) aqueles que não concordaram em participar da pesquisa e não assinaram o TCLE. A amostra inicial foi composta por 81 funcionários distribuídos nas categorias: auxiliares de limpeza, técnicos de laboratórios, técnicos administrativos, vigilantes, porteiros, motoristas e secretários. Após considerados os critérios de exclusão, 39 funcionários não se enquadraram na pesquisa e ao final 42 funcionários participaram da pesquisa. O contato com os funcionários foi feito em dois momentos, o primeiro teve como objetivo explicar os objetivos, bem como os procedimentos para coleta dos dados da pesquisa e solicitar a leitura e assinatura do termo de consentimento livre e esclarecido (TCLE). Em seguida, foram coletados os dados antropométricos e demais informações necessárias ao preenchimento da ficha clínica.

A coleta dos dados foi realizada entre novembro e dezembro de 2018, utilizando-se como instrumento uma ficha para preenchimento dos dados clínicos e antropométricos como peso, altura, Índice de massa corporal (IMC), Circunferência da Cintura (CC), Circunferência do quadril (CQ), Relação Cintura Quadril (RCQ) e Relação Cintura-Estatura (RCE), além disso foram coletados dados referentes à 
histórico familiar de doenças crônicas e dados pessoais, como idade, renda e escolaridade, complementados pelos dados comportamentais como hábito de fumar, consumo de bebidas alcoólicas e prática de atividade física.

O IMC foi obtido pela fórmula IMC = peso $(\mathrm{kg}) /$ altura2 $(\mathrm{m})$, e classificado de acordo com os valores estabelecidos para adultos pela World Health Organization (WHO). O peso foi aferido por balança portátil Tanita BC533® (Brasil), com o avaliado em pé e descalço. A altura foi medida por estadiomêtro Altura exata® (Brasil), com o indivíduo em pé, descalço, com os calcanhares juntos, costas retas e os braços estendidos ao lado do corpo. As medidas de circunferência foram aferidas com uma trena antropométrica metálica, da marca SANNY. A CC foi medida da distância entre a crista ilíaca e o rebordo costal inferior e foram classificadas como circunferência da cintura aumentada, aquelas que apresentaram valores $>102 \mathrm{~cm}$ para homens e $>88 \mathrm{~cm}$ para mulheres, de acordo com parâmetros do protocolo NCEPIATP III (2002). A CQ foi aferida na região de maior perímetro entre a cintura e a coxa, a RCQ foi calculada por meio da razão entre $C C$ e $C Q(C C / C Q)$ e classificada de acordo com os pontos de corte da WHO, a RCE foi calculada a partir da razão entre CC e altura (CC/Altura), ambas em centímetros, considerando valores $\geq 0,5$ para homens e mulheres, como risco. Após a coleta os dados foram agrupados, tabulados e analisados. Os dados foram apresentados em gráficos e tabelas, apresentados em valores de média e percentuais, utilizando o programa Microsoft Office Exce|® 2016, com análise através de estatística descritiva.

O projeto de pesquisa foi submetido ao Comitê de Ética em Pesquisa (CEP) da Faculdade Santa Maria, e foi aprovado sob número de protocolo $\mathrm{n}^{\circ}$ 3.000.671. Todos os funcionários participantes do estudo foram apresentados ao TCLE, e após leitura foram convidados a participar da pesquisa. Assim, ficou autorizada a participação e publicação dos resultados em anonimato. 


\section{RESULTADOS E DISCUSSÕES}

Entre os 42 funcionários que participaram da pesquisa, 15 eram homens (36\%) e 27 mulheres (64\%), dentre eles, 8 homens apresentavam faixa etária entre 30 e 39 anos e 9 mulheres entre 40 e 49 anos. Em relação ao grau de escolaridade, entre os homens, 8 relataram ter ensino médio completo. Quanto às mulheres, 10 relataram terem ensino médio completo e 10 ensino superior completo. Em relação à renda salarial, 9 homens e 16 mulheres afirmaram receber 2 salários mínimos. Os dados estão apresentados na Tabela 1.

O presente estudo também avaliou hábitos comportamentais que estão relacionados ao aumento do risco cardiometabólico em homens e mulheres, como o consumo de álcool, o tabagismo e a prática de atividade física. Conforme disposto na Tabela 2, observou-se que entre as mulheres, 9 relataram praticar algum tipo de atividade física regularmente, 13 relataram consumir álcool e 4 fazem uso de cigarros. Entre os homens, 7 afirmaram praticar atividade física regularmente, 9 consomem álcool e nenhum deles relatou fazer uso de cigarros.

Segundo Carvalho, Dutra e Araújo (2009), a gênese da obesidade e da SM está associada a diversos fatores, como genéticos, ambientais, socioculturais e comportamentais. Em adição, de acordo Simão et al. (2013), a falta de exercício físico regular, o tabagismo, o alcoolismo e a má alimentação, constituem-se como fatores de riscos modificáveis e quando corrigidos reduzem consideravelmente as chances de eventos cardiovasculares. 
Tabela 1 - Caracterização da amostra segundo a faixa etária, grau de escolaridade e renda salarial.

\begin{tabular}{|c|c|c|}
\hline GÊNERO & MASCULINO 15 & FEMININO 27 \\
\hline \multicolumn{3}{|c|}{ FAIXA ETÁRIA } \\
\hline $20-29$ & 3 & 7 \\
\hline $30-39$ & 8 & 6 \\
\hline $40-49$ & 2 & 9 \\
\hline $50-60$ & 2 & 5 \\
\hline \multicolumn{3}{|c|}{ ESCOLARIDADE } \\
\hline ENSINO FUNDAMENTAL & 4 & 7 \\
\hline ENSINO MÉDIO & 8 & 10 \\
\hline ENSINO SUPERIOR/TÉCNICO & 3 & 10 \\
\hline \multicolumn{3}{|c|}{ RENDA SALARIAL } \\
\hline 1 SALÁRIO & 4 & 5 \\
\hline 2 SALÁRIOS & 9 & 16 \\
\hline >3 SALÁRIOS & 2 & 6 \\
\hline
\end{tabular}

Fonte: Dados da pesquisa, 2018.

Tabela 2 - Fatores de risco não antropométricos, em ambos sexos.

\begin{tabular}{l|cc}
\hline ATIVIDADE FÍSICA & 7 & 9 \\
CONSUMO DE ALCOOL & 9 & 13 \\
USO DE CIGARRO & 0 & 4
\end{tabular}

Fonte: Dados da pesquisa, 2018.

De acordo com Borba, Lemos e Hayasida (2015), o uso excessivo de álcool ocasiona problemas no músculo cardíaco, que por sua vez acarreta inúmeros distúrbios cardiovasculares. Observou-se que o hábito de fumar também está relacionado a eventos cardiovasculares como IAM e AVC, afetando principalmente mulheres, sobretudo, aquelas que iniciaram o uso do cigarro antes dos 16 anos de idade. O estudo de Teston et al. (2015) buscou identificar fatores de risco para as DCVs em adultos da região metropolitana de Maringá-PR, e demonstraram que 40,8\% consumiam bebidas alcoólicas, $54,5 \%$ se alimentavam de forma inadequada, pois apresentaram baixo consumo de frutas e/ou hortaliças e/ou verduras ( $<5$ vezes/ semana) e $82,8 \%$ foi classificada como sedentária. O estudo também concluiu que o risco de ocorrência de DCV estava relacionado ao consumo de álcool e alterações 
nas medidas antropométricas.A avaliação do risco cardiometabólico, com base nas medidas antropométricas de Índice de Massa Corporal (IMC), Circunferência da Cintura (CC), Relação Cintura Quadril (RCQ) e Relação Cintura-Estatura (RCE), deu-se por meio da comparação entre as médias encontradas para os participantes do estudo e os respectivos pontos de corte. Em relação ao IMC, constatou- se que 14 mulheres foram classificadas como obesas, sendo 4 obesas grau III, 3 obesas grau II e 7 obesas grau I. Ainda no grupo das mulheres, verificou-se que 7 estavam com sobrepeso e 6 eutróficas. No grupo dos homens, 4 foram classificados com obesidade grau I, 8 com sobrepeso e 3 estavam eutróficos. Nenhum participante apresentou obesidade grau II e III. Os dados podem ser observados na Figura 1.

Figura 1 - Classificação do Estado Nutricional, segundo o IMC, em ambos sexos.

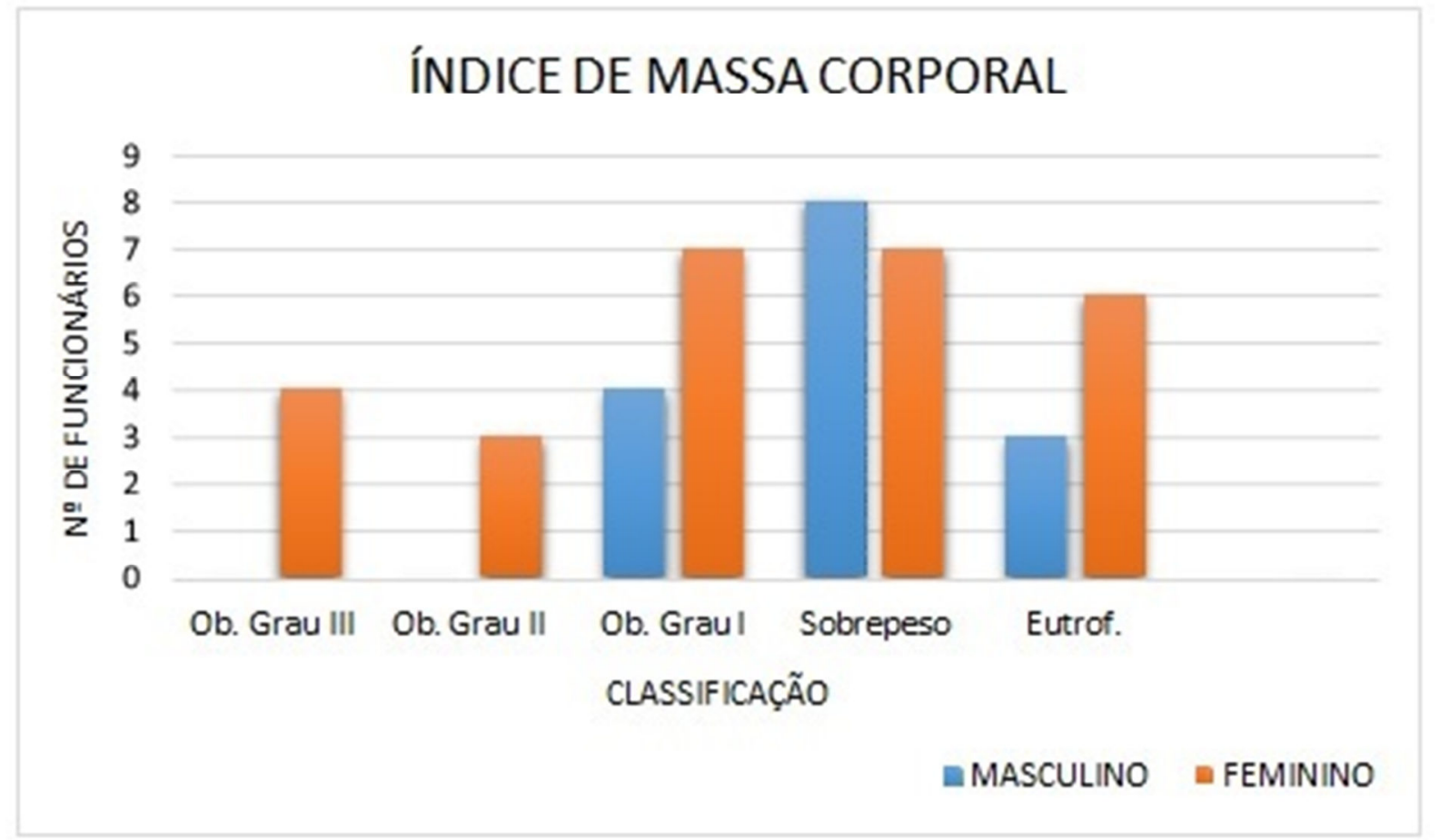

Fonte: Dados da pesquisa, 2018.

Segundo Kamimura, Sampaio e Cuppari (2009) o indicador antropométrico mais utilizado é o IMC ou também chamado Índice de Quetelet, considerado um parâmetro simples para avaliar a gordura corpórea e o risco de morbimortalidade. Contudo, apresenta algumas limitações, a principal delas é a incapacidade de distinguir a massa magra da gordura corpórea, como também não consegue 
identificar a forma como essa gordura está distribuída. Nesse sentido, recomenda-se que o IMC seja utilizado associado a parâmetros que meçam a composição e a distribuição da gordura corpórea.

De acordo com os dados da pesquisa $51,8 \%$ das mulheres e $26,6 \%$ dos homens foram classificados como obesos, resultado que sugere uma maior prevalência de obesidade entre as mulheres, no entanto, o fato da amostra ser composta principalmente por mulheres pode aumentar as chances de se ter um maior número de pessoas obesas. Este achado corrobora com os resultados encontrados por Géa-horta et al. (2018), que avaliou os efeitos das mudanças nas mediadas antropométricas em 387 moradores da zona rural do Brasil, tendo como principal parâmetro de avaliação o IMC. A pesquisa demonstrou que as mulheres apresentaram IMC maior em comparação aos homens e a obesidade foi cinco vezes mais prevalente nas mulheres. Além disso, verificou-se que o aumento do IMC e da CC são preditores relevantes para o aumento da pressão arterial diastólica e alterações no perfil lipídico.

A CC é uma medida bastante utilizada para avaliar a distribuição da gordura corporal. O excesso de gordura na região abdominal, assim como a proporção da sua distribuição pode ser um importante fator predisponente de distúrbios metabólicos associados à obesidade e à SM, como as DCVs, os pontos de cortes utilizados são de $102 \mathrm{~cm}$ para homens e de $88 \mathrm{~cm}$ para mulheres, representando um fator de risco independente à doença cardiovascular (NCEP, 2002; LITCHFORD, 2018).

No presente estudo, os dados de CC apresentaram-se elevados em ambos sexos, sobretudo no sexo feminino. Verificou-se que 15 mulheres e 5 homens apresentaram risco muito elevado, 5 mulheres e 4 homens apresentaram risco elevado com base na medida de CC, conforme observado na Figura 2. 
Figura 2 - Classificação do risco cardiovascular, segundo a CC, em ambos sexos.



Fonte: Dados da pesquisa, 2018.

Com base nos dados de CC é possível constatar que $21,4 \%$ dos participantes da pesquisa apresentaram risco elevado de desenvolvimento de DCV e 47,6\% apresentaram risco muito elevado. Além disso, 55,5\% das mulheres apresentam risco muito elevado para DCV. Um estudo realizado por Sampaio et al (2017), avaliou o risco cardiometabólico de 91 usuários do Sistema Único de Saúde por meio de medidas antropométricas, observou-se que, em relação à $\mathrm{CC}, 62$ usuários $(68,1 \%)$ apresentavam risco elevado e apenas 29 usuários $(31,9 \%)$ estavam fora de risco, demonstrando uma maior prevalência de indivíduos em risco de desenvolver DCV.

Outro estudo realizado com trabalhadores agroindustriais e produtores rurais, sendo 87 mulheres $(62,6 \%)$ e 52 homens $(37,4 \%)$, demonstrou que $22,3 \%$ dos participantes apresentaram risco elevado e $47,5 \%$ apresentaram risco muito elevado, segundo a CC, além disso, demonstrou que entre as mulheres, $64,4 \%$ apresentaram risco muito elevado. Adicionalmente, os dados do estudo também demonstraram 
que $48,3 \%$ da população apresentava risco elevado, segundo a RCQ (SANTOS et al., 2019).

Segundo ABESO (2016), a RCQ foi uma das primeiras medidas a serem utilizadas para avaliação da obesidade central e distribuição de gordura corporal e está frequentemente associada ao risco de comorbidades, inclusive o risco cardiometabólico. No presente estudo, as medidas de RCQ foram classificadas de acordo com os pontos de corte estabelecidos pela WHO para a faixa etária de 20 a 59 anos, para homens: risco baixo $(<0,83-<0,90)$, risco moderado $(0,83-0,98)$, risco alto $(0,89-1,02)$ e risco muito alto $(>0,94->1,02)$ e para as mulheres: risco baixo $(<0,71-<0,74)$, risco moderado $(0,71-0,81)$, risco alto $(0,76-0,88)$ e risco muito alto $(>0,82$ - >0,88). A classificação da $R C Q$, segundo o sexo, está disposto na Figura 3.

Figura 3 - Classificação do risco cardiometabólico, segundo a RCQ, em ambos sexos.

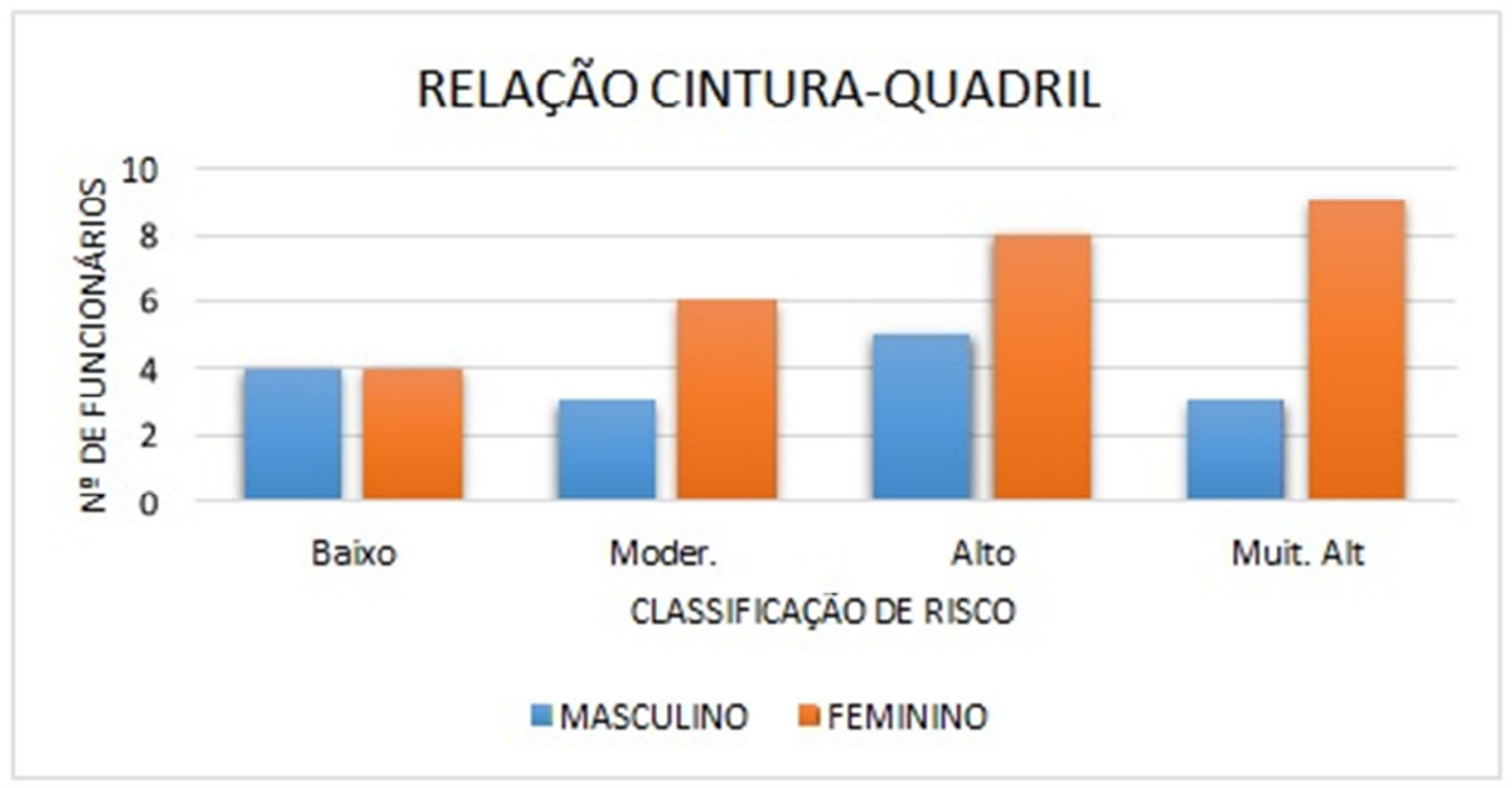

Fonte: Dados da pesquisa, 2018.

Pode-se identificar que, com base nas medidas de RCQ, 12 funcionários $(28,5 \%)$ apresentaram risco muito alto, 13 (30,9\%) apresentaram risco alto, $9(21,4 \%)$ risco moderado e $8(19,2 \%)$ baixo risco. Além disso, observou-se que 25 
participantes $(59,5 \%)$ foram classificados em risco alto e muito alto, o que sugere elevado risco cardiometabólico para a maioria dos funcionários participantes da pesquisa. Estudo semelhante, realizado por Porto et al. (2016) analisou algumas medidas antropométricas relacionadas à adiposidade em funcionários de um hospital universitário, demonstrando que dos 380 participantes, 214 (56,3\%) apresentavamse dentro dos parâmetros normais e 166 (43,7\%) apresentavam risco elevado, com base na RCQ.

Em estudo semelhante que analisou o risco cardiovascular e o estresse ocupacional em 45 trabalhadores da área da saúde, observou-se que $73,4 \%$ dos funcionários apresentavam risco moderado e alto risco, segundo a $\mathrm{RCQ}$. Além disso, foi visto que 28 funcionários $(62,2 \%)$ estavam com percentual de gordura acima do recomendado e 27 (60\%) apresentaram sedentarismo, fatores que sugerem uma tendência elevada para obesidade (ULGUIM et al., 2019).

De acordo com Corrêa et al. (2017), outra medida importante para verificar a adiposidade central é a RCE, pois está fortemente associada ao risco cardiometabólico e a alta mortalidade, independente do peso corporal. A atual pesquisa utilizou a medida de $\mathrm{RCE} \geq 0,5$ como fator de risco para DCV, em ambos sexos, conforme validado o referido ponto de corte. Observou-se que 20 mulheres e 11 homens foram classificados como grupo de risco, segundo a RCE, conforme demonstrado na Tabela 3.

Tabela 3 - Comparação dos pontos de corte da RCE entre os sexos.

\begin{tabular}{l|cc}
\hline PONTOS DE CORTE & $\mathbf{N}^{\circ}$ HOMENS & $\mathbf{N}^{\circ}$ MULHERES \\
\hline$<0,5$ (NÃO APRESENTA RISCO) & 4 & 7 \\
$\geq 0,5$ (APRESENTA RISCO) & 11 & 20 \\
\hline
\end{tabular}

Fonte: Dados da pesquisa, 2018.

De acordo com os dados da relação cintura/estatura, 31 funcionários $(73,8 \%)$ apresentaram risco, com proporções semelhantes para ambos sexos. Resultados semelhantes foram evidenciados no estudo de Bolzan e Mussoi (2017), que avaliou 120 prontuários de uma clínica-escola de nutrição a fim de verificar o risco de doenças cardiovasculares através de medidas antropométricas. De acordo com os achados, foi visto que a média de RCE permaneceu acima do ponto de corte 
recomendado, tanto em homens como em mulheres, sugerindo que $91 \%$ dos pacientes apresentavam risco. Em adição, percebeu-se que a RCE e o Índice de Conicidade (IC) demonstrou maior sensibilidade em detectar $\mathrm{O}$ risco cardiometabólico comparado ao IMC, CC e RCQ.

O estudo de Cardozo et al. (2018) avaliou o estado nutricional de 24 trabalhadores de uma unidade de alimentação e nutrição (UAN), dos quais 15 $(62,5 \%)$ eram mulheres e $9(37,5 \%)$ eram homens. Foi visto que em relação a RCE, 8 colaboradores $(33,3 \%)$ estavam fora de risco e 16 colaboradores $(66,6 \%)$ apresentaram risco. Além do mais, viu-se que comparado com as outras medidas antropométricas (IMC, CC e RCQ), a RCE novamente demonstrou maior sensibilidade, identificando possível risco cardiometabólico na maioria dos trabalhadores.

\section{CONCLUSÃO}

Os resultados revelaram elevado risco cardiometabólico, com base nas medidas antropométricas, dos funcionários participantes do estudo, evidenciado pelos parâmetros de IMC, CC, RCQ e RCE, sobretudo, na população feminina que representou a maior parte da amostra. Dessa forma, é possível concluir que estes parâmetros antropométricos são relevantes no rastreio do risco cardiovascular e quando associado a outros fatores, como hábitos de vida, podem predizer a condição de saúde de uma população e assim fomentar estratégias de intervenções nutricionais que reduzam o risco associado ao excesso de gordura corporal, evidenciado pelas medidas antropométricas, e assim melhorar a condição nutricional e qualidade de vida da população. 


\section{REFERÊNCIAS BIBLIOGRÁFICAS}

BARROSO, T. A. et al. Association of Central Obesity with The Incidence of Cardiovascular Diseases and Risk Factors. International Journal Of Cardiovascular Sciences, v. 30, n. 5 , p.416-424, 2017.

BOLZAN, A.; MUSSOI, T. D. Prevalência De Risco Cardiovascular De Acordo Com Os Indicadores Antropométricos Convencionais E Não Convencionais. Disciplinarum Scientia, v. 17, n. 3, p.353-360, 2017.

BORBA, C. S.; LEMOS, I. G. S.; HAYASIDA, N. M. de A. Epidemiologia e Fatores de Risco Cardiovasculares em Jovens Adultos: Revisão da Literatura. Revista Saúde e Desenvolvimento Humano, v. 3, n. 31, p.52-60, 2015.

CARDOZO, Jean da Silva et al. AVALIAÇÃO DO ESTADO NUTRICIONAL DE FUNCIONÁRIOS DE UNIDADES DE ALIMENTAÇẪO DO VALE DO SÃO FRANCISCO. Revista Brasileira de Obesidade, Nutrição e Emagrecimento, v. 12, n. 76, p.1050-1055, 2018.

CARVALHO, K. M. B. de; DUTRA, E. S.; ARAÚJO, M. S. M. OBESIDADE E SÍNDROME.

METABÓLICA. In: CUPPARI, Lilian (Org.). Nutrição: nas doenças crônicas não- transmissíveis. Barueri: Manole, 2009. Cap. 3. p. 91-138.

CORRÊA, M. M. et al. Razão cintura-estatura como marcador antropométrico de excesso de peso em idosos brasileiros. Cadernos de Saúde Pública, v. 33, n. 5, p.1-14, 2017.

GÉA-HORTA, T. et al. Anthropometric changes and their effects on cardiometabolic risk factors in rural populations in Brazil. Ciência \& Saúde Coletiva, v. 23, n. 5, p.1415-1423, 2018.

KAMIMURA, M. A.; SAMPAIO, L. R.; CUPPARI, L. Avaliação Nutricional da Prática Clínica. In: CUPPARI, Lilian (Org.). Nutrição: nas doenças crônicas não-transmissíveis. Barueri: Manole, 2009. Cap. 2. p. 27-59.

LITCHFORD, M. D. Clínica: Avaliação Bioquímica, Física e Funcional. In: MAHAN, L. Kathleen; RAYMOND, Janice L. Krause: Alimentos, Nutrição e Dietoterapia. 14. ed. Rio de Janeiro: Elsevier, 2018. Cap. 7. p. 393-473.

LYSEN, Lucinda K.; ISRAEL, Donna A. Nutrição no Controle da Massa Corporal. In: MAHAN, L. Kathleen; RAYMOND, Janice L. Krause: Alimentos, Nutrição e Dietoterapia. 14. ed. Rio de Janeiro: Elsevier, 2018. Cap. 21. p. 1432-1512.

MARTINI, F. A. N; BORGES, M. B; GUEDES, D. P. Hábito alimentar e síndrome metabólica em uma amostra de adultos brasileiros. 2014. Archivos Latino Americanos de Nutrición, [s.I], v. 64, n. 3, p. 161-173, 2014.

NCEP. Third Report of the National Cholesterol Education Program (NCEP) Expert Panel on Detection, Evaluation, and Treatment of High Blood Cholesterol in Adults (Adult Treatment Panel III) Final Report. Circulation, v. 106, n. 25, p.3227-3234, 2002.

ORGANIZAÇÃO PAN-AMERICANA DE SAÚDE (OPAS) (Brasil). Organização Mundial de Saúde (OMS) (Org.). Doenças cardiovasculares. 2017. Disponível em: $<$ https://www.paho.org/bra/index.php?option=com_content\&view=article\&id=5253:doencascardiovasculares\&ltemid=1096>. Acesso em: 12 fev. 2020. 
PORTO, D. B. et al. Autopercepção de saúde em trabalhadores de um Hospital Universitário e sua associação com indicadores de adiposidade, pressão arterial e prática de atividade física. Ciência \& Saúde Coletiva, v. 21, n. 4, p.1113-1122, 2016.

RAYMOND, Janice L.; COUCH, Sarah C. Dietoterapia para Doença Cardiovascular. In: MAHAN, L. Kathleen; RAYMOND, Janice L. Krause: Alimentos, Nutrição e Dietoterapia. 14. ed. Rio de Janeiro: Elsevier, 2018. Cap. 33. p. 2402-2516.

STEVENS, B. et al. The Economic Burden of Heart Conditions in Brazil. Arquivos Brasileiros de Cardiologia, p.1-8, 2018. Sociedade Brasileira de Cardiologia.

SANTOS, P. R. dos et al. PARÂMETROS BIOQUÍMICOS E ANTROPOMÉTRICOS DE TRABALHADORES RURAIS: ESTUDO DE NOVAS FERRAMENTAS ANALÍTICAS COM SUPERVISÃO DE TÉCNICAS PADRÃO. Revista Brasileira de Obesidade, Nutrição e Emagrecimento, v. 13, n. 80, p.478-487, 2019.

SIMÃO, Af et al. I Diretriz Brasileira de Prevenção Cardiovascular. Arquivos Brasileiros de Cardiologia, v. 101, n. 6, p.1-63, 2013.

SOUSA, D. S. de; SOUZA, A. E. S. de; RIBEIRO, K. A. S. Avaliação do risco coronariano de acordo com a relação cintura-quadril em universitários. Revista Brasileira de Fisiologia do Exercício, v. 17, n. 1, p.1-7, 2018.

TESTON, E. F. et al. Fatores associados às doenças cardiovasculares em adultos. Revista USP, v. 49, n. 2, p.95-102, 2015.

ULGUIM, F. O. et al. Trabalhadores da saúde: risco cardiovascular e estresse ocupacional. Revista Brasileira de Medicina do Trabalho, v. 17, n. 1, p. 61-68, 2019. 